Check for updates

Cite this: RSC Adv., 2017, 7, 40218

Received 30th June 2017

Accepted 9th August 2017

DOI: $10.1039 / c 7 r a 07264 c$

rsc.li/rsc-advances

\section{Improving the rates of Pd-catalyzed reactions by exciting the surface plasmons of AuPd bimetallic nanotriangles}

\author{
Mahesh K. Gangishetty, ${ }^{a}$ Adriana M. Fontes, ${ }^{\mathrm{b}}$ Marcos Malta, ${ }^{\mathrm{b}}$ Timothy L. Kelly ${ }^{\star a}$ \\ and Robert W. J. Scott (iD)*a
}

Gold nanoparticles exhibit unique optical properties due to surface plasmon oscillations when they interact with light. By utilizing their optical properties, the rates of many chemical reactions have been improved in the presence of visible light. The properties of plasmonic nanoparticles are highly tunable based on the size and shape of the nanoparticle. Here, we have used anisotropic AuPd bimetallic nanotriangles to improve the rates of $\mathrm{Pd}$-catalyzed reactions in the presence of visible light. We synthesized AuPd core-shell bimetallic nanotriangles and performed Suzuki cross-coupling and hydrogenation reactions in light and dark conditions. Upon illuminating AuPd nanotriangles with an array of green LEDs (power $\sim 500 \mathrm{~mW}$ ), enhanced catalytic activity of palladium was observed. In order to understand the relative contributions of individual plasmonic effects, such as plasmonic hot electron transfer and plasmonic heating effects, the reaction temperatures were monitored, and careful control experiments were run at different temperatures. Our results indicated that the enhancement in the rate of these Pd-catalyzed reactions is primarily due to the plasmonic heating effect.

\section{Introduction}

Plasmonic nanoparticles have been extensively studied as light harvesting materials in various applications such as photovoltaics, ${ }^{1-4}$ biotechnology, ${ }^{5}$ catalysis, ${ }^{6}$ and surface-enhanced Raman spectroscopy. ${ }^{7}$ In particular, employing their light trapping properties to drive chemical transformations is an emerging field. ${ }^{6}$ Upon interacting with certain frequencies of light, metal nanoparticles show unique optical properties due to the collective oscillation of their conduction band electrons. This phenomenon is called localized surface plasmon resonance. This phenomenon has also been observed in some metal oxides such as $\mathrm{WO}_{x}{ }^{8}$ and $\mathrm{Ce}: \mathrm{In}_{2} \mathrm{O}_{3}{ }^{9}{ }^{9}$ The optical characteristics of plasmonic nanoparticles are tunable based on the size and the shape of the nanoparticle. ${ }^{10}$ The energy accumulated in these oscillating surface plasmons can be used in several ways to accelerate the rates of chemical reactions. This energy can be either directly used to drive a chemical reaction, ${ }^{6,11}$ or it can be transferred to an existing catalyst such as a semiconducting metal oxide (e.g., $\mathrm{TiO}_{2}, \mathrm{CeO}_{2}$ ) or a metal (e.g., $\mathrm{Cu}, \mathrm{Pd}$, and $\mathrm{Ni}$ ) nanoparticle. ${ }^{4,12-15}$ Among these catalysts, enhancing the activity of Pd nanoparticles using solar energy is attractive since Pd nanoparticles are known to catalyze many organic reactions,

${ }^{a}$ Department of Chemistry, University of Saskatchewan, 110 Science Place, Saskatoon, SK, S7N 5C9, Canada. E-mail: robert.scott@usask.ca; tim.kelly@usask.ca

${ }^{b}$ Instituto de Química, Universidade Federal da Bahia, Campus Ondina, Salvador, BA, Brazil including $\mathrm{C}-\mathrm{C}$ bond forming reactions. Also, heterogeneous Pd catalysts can be easily recovered from the reaction mixture and reused for several reaction cycles. Recently, by using plasmonic nanoparticles, the rates of Pd-catalyzed reactions such as crosscoupling, ${ }^{16,17}$ hydrogenation, ${ }^{18-21}$ and oxidation reaction ${ }^{22-24}$ have been improved, and it has been found that bimetallic nanoparticles show superior activity compared to the pure metallic nanoparticles. ${ }^{25,26}$ The most studied bimetallic catalysts in plasmon-enhanced Pd-catalyzed reactions are AuPd bimetallic nanoparticles. Here, the Au/Pd ratio has been shown to be critical for both preserving the Au plasmonic properties as well as the catalytic activity of Pd. ${ }^{26}$ In addition to this, the power of the incoming light also plays a key role in the plasmonic enhancement. ${ }^{17,26}$

It has been demonstrated that two plasmonic effects contribute to the enhancement of Pd-catalytic activity in AuPd bimetallic nanoparticle systems: plasmonic local heating, and plasmonic hot electron transfer. ${ }^{16,17,26}$ After the excitation of surface plasmons, they can decay non-radiatively via generation of hot electrons in the metal nanoparticles. The energy of these hot electrons is either redistributed among the ground state electrons through electron-electron scattering or dissipated in the form of heat by electron-phonon scattering., ${ }^{4,27,28}$ The heat generated by the metal nanoparticles can increase the temperature at their surface up to $500{ }^{\circ} \mathrm{C} .{ }^{6}$ In a colloidal solution of metal nanoparticles, this local heat dissipates into the solution and increases the overall temperature of the bulk solution. This plasmonic photothermal effect was found to accelerate the rates 
of chemical reactions when $\mathrm{Au} / \mathrm{ZnO},{ }^{29} \mathrm{Au} / \mathrm{Fe}_{2} \mathrm{O}_{3},{ }^{30}$ and bimetallic AuPd nanoparticles ${ }^{17,21,31,32}$ were employed as catalysts. In addition to the plasmonic heating effect, some reports on AuPd catalyzed reactions also observed a contribution from plasmonic hot electron transfer from Au to Pd in improving the reaction rates. ${ }^{26,33}$ It has been demonstrated that in AuPd nanoparticles, the Fermi energy levels of $\mathrm{Au}$ and $\mathrm{Pd}$ are close to each other ( $\mathrm{Pd}$ is $5.0 \mathrm{eV}$, and $\mathrm{Au}$ is $4.7 \mathrm{eV}){ }^{34}$ Upon exciting the surface plasmons in $\mathrm{Au}$, the hot electrons can easily be transferred from $\mathrm{Au}$ to $\mathrm{Pd}$. As a result of this transfer, the number of active catalytic sites on Pd increases, and this improves the overall rate of the reaction. ${ }^{16}$ Such hot electron transfer from $\mathrm{Au}$ to Pd was described by recording a single particle photoluminescence (PL) spectra from an $\mathrm{Au}-\mathrm{Pd}$ nanorod and an $\mathrm{Au}$ nanorod. ${ }^{35}$ The authors observed a decrease in the PL intensity for the AuPd nanorod compared to the Au nanorod, which was attributed to hot electron transfer from Au and Pd. Further, by using a $\mathrm{TiO}_{2}$ spacer between Au to Pd in an AuPd nanoparticle, Wang et al. controlled the electron transfer from Au to Pd. This resulted in a decrease in the conversion of a Suzuki reaction compared to AuPd nanoparticles without any spacer. ${ }^{17}$ However, the difference in conversion was very low, and it also depended on the power of the incoming light. Another study, with the help of DFT calculations, reported that hot electrons generated in AuPd nanoparticles are directly transferred to the adsorbed molecules and drive the chemical transformation. ${ }^{16}$

All these plasmonic effects are highly dependent on the power of the incoming light as well as on the morphology of the metal nanoparticle. Various morphologies of Au and Ag nanoparticles such as nanorods, ${ }^{17,35,36}$ nanowheels, ${ }^{32}$ nanocages ${ }^{21}$ and spherical nanoparticles ${ }^{16,20,26,37}$ have been used to improve the catalytic activity of palladium. Here, we employed plasmonic Au nanotriangles to harvest light in order to photophysically improve the rates of Pd-catalyzed reactions. Due to the presence of sharp features, the nanotriangles are expected to show strong plasmonic effects compared to other shapes. ${ }^{38,39}$ Also, due to the plate-like morphology of a nanotriangle, the number of active sites (i.e., surface area) available for catalytic reactions to take place is higher compared to a spherical nanoparticle. By using AuPd bimetallic nanotriangles, we performed Suzuki cross-coupling and hydrogenation reactions in both dark and light conditions. The reactions performed in the light showed higher conversions compared to the ones carried out in the dark. The mechanism of this plasmonic enhancement was investigated by performing several control experiments. Our results indicate that the enhancements in the rates of both Suzuki and hydrogenation reactions upon illumination are mainly due to plasmonic heating effects. Further stability studies show that the sharp-featured triangles are unstable, and after two cycles of Suzuki reactions their catalytic activity is severely compromised.

\section{Experimental section}

\section{Materials}

Hydrogen tetrachloroaurate(III) (99\%), 4-iodobenzoic acid, and 2-methyl-3-buten-2-ol (MBE, 98\%) were purchased from Sigma-
Aldrich. Potassium tetrachloropalladate (99\%), cetyltrimethylammonium chloride (CTAC, 96\%) and all the boronic acids used for Suzuki reactions were purchased from Alfa Aesar. Potassium iodide and sodium hydroxide were purchased from Fisher Scientific. All chemicals were used directly without any further purification, and aqueous solutions were prepared using Milli-Q water (18.2 M $\mathrm{cm})$.

\section{Characterization}

Transmission electron microscopy (TEM) images were collected using a HT7700 TEM operating at $100 \mathrm{kV}$. TEM samples were prepared by drop casting nanoparticle solutions onto a carboncoated 300 mesh Cu TEM grid (Electron Microscopy Sciences). UV-vis spectra were measured using a Varian Cary 50 Bio UV-vis spectrophotometer with an optical path length of $1 \mathrm{~cm} .{ }^{1} \mathrm{H}$ NMR spectra were collected by using a Bruker Avance $500 \mathrm{MHz}$ spectrometer.

\section{Methods}

Synthesis of Au nanotriangles. Au nanotriangles were synthesized by following a literature procedure. ${ }^{40}$ Briefly, aqueous CTAC (16.0 mL, 0.10 M) was dissolved in $20.0 \mathrm{~mL}$ Milli$\mathrm{Q}$ water in a $250 \mathrm{~mL}$ Erlenmeyer flask. To this solution, aqueous solutions of $\mathrm{KI}(0.75 \mathrm{~mL}, 0.1 \mathrm{M}), \mathrm{HAuCl}_{4}(0.80 \mathrm{~mL}, 25.4 \mathrm{mM})$ and $\mathrm{NaOH}(0.20 \mathrm{~mL}, 0.10 \mathrm{M})$ were added sequentially. At each addition, the solution was stirred manually by shaking the flask. Aqueous ascorbic acid $(1.0 \mathrm{~mL}, 0.064 \mathrm{M})$ was then added, and then immediately, an aqueous $\mathrm{NaOH}(0.20 \mathrm{~mL} 0.10 \mathrm{M})$ solution was rapidly injected into the reaction mixture, while simultaneously shaking the flask. Finally, the entire mixture was kept undisturbed for 15 min until the solution became dark blue in color. This dark blue solution was centrifuged at $5500 \mathrm{rpm}$ (4194 g) for $30 \mathrm{~min}$, and then the supernatant was decanted. The Au nanotriangles were then redispersed in water.

Synthesis of AuPd nanotriangles. A concentrated Au nanotriangle solution $(15 \mathrm{~mL}$, optical density $=4.5$ at $610 \mathrm{~nm})$ was dispersed in aqueous CTAC ( $30 \mathrm{~mL}, 0.010 \mathrm{M})$ in an Erlenmeyer flask. To this mixture, an aqueous $\mathrm{K}_{2} \mathrm{PdCl}_{4}(2.0 \mathrm{~mL}, 0.010 \mathrm{M})$ solution was added while stirring the solution, which was maintained at $40{ }^{\circ} \mathrm{C}$ using a hot water bath. After $10 \mathrm{~min}$ of stirring, aqueous ascorbic acid $(2.0 \mathrm{~mL}, 0.040 \mathrm{M})$ was added, the flask was covered and the stirring was continued for $8 \mathrm{~h}$ at $40^{\circ} \mathrm{C}$ by covering the flask with parafilm. Finally, this solution was centrifuged at $5500 \mathrm{rpm}(4194 \mathrm{~g})$ for $30 \mathrm{~min}$, and the AuPd bimetallic nanotriangles were collected by decanting the supernatant. The AuPd nanotriangles were then dispersed in water.

\section{Catalytic reactions}

Suzuki cross-coupling reactions. In a model cross-coupling reaction, $0.5 \mathrm{mmol}$ of $p$-iodobenzoic acid and $0.6 \mathrm{mmol}$ of phenylboronic acid were dissolved in a mixture of $8.0 \mathrm{~mL}$ of Milli-Q water and $2 \mathrm{~mL}$ of ethanol. To this mixture, $2.0 \mathrm{mmol}$ of $\mathrm{K}_{2} \mathrm{CO}_{3}$ dissolved in $2.0 \mathrm{~mL}$ of water was added. The solution was mixed well to dissolve all of the reactants. Next, $2.0 \mathrm{~mL}$ of the as-synthesized AuPd nanotriangle solution (optical density = 
4.5 at $532 \mathrm{~nm}$ ) was added. The final optical density of the nanotriangles in the resulting solution was $\sim 0.75$ at $532 \mathrm{~nm}$. This reaction was performed both with and without light illumination using a home-made LED source (Luxeon), which consisted of 8 green LEDs which have a maximum emission wavelength of $530 \mathrm{~nm}$ and a emission range between 450 and $600 \mathrm{~nm}$ (power $\sim 500 \mathrm{~mW}$ ). At each hour, $200 \mu \mathrm{L}$ aliquots of the reaction mixture were collected, the reaction was quenched with $200 \mu \mathrm{L}$ of concentrated $\mathrm{H}_{2} \mathrm{SO}_{4}$, and the products extracted with dichloromethane. The solvent was evaporated in a stream of air, and the leftover solids were dissolved in $\mathrm{CDCl}_{3}$ and characterized by ${ }^{1} \mathrm{H}$ NMR. The rest of the cross-coupling reactions were performed in a similar way except for the number of moles of $\mathrm{K}_{2} \mathrm{CO}_{3}$ in the reaction mixture. For reactants containing an acid functional group, we used $2.0 \mathrm{mmol}$ of $\mathrm{K}_{2} \mathrm{CO}_{3} ; 1.0 \mathrm{mmol}$ of $\mathrm{K}_{2} \mathrm{CO}_{3}$ was used otherwise. Also, the concentrated $\mathrm{H}_{2} \mathrm{SO}_{4}$ was added only when the reactants contained carboxylic groups, otherwise the reaction mixture was directly extracted into dichloromethane.

Hydrogenation of 2-methyl-3-buten-2-ol. In a $1 \mathrm{~L}$ round bottom flask, $3.0 \mathrm{~mL}$ of AuPd nanotriangle solution (optical density 0.75 at $532 \mathrm{~nm}$ ) was added to $6 \mathrm{~mL}$ Milli-Q water. To this mixture, $150 \mu \mathrm{L}$ of methanol was added and then the flask was sealed tight using a septum. The flask was then purged with hydrogen gas for 20 minutes; the flask was then filled with $\mathrm{H}_{2}$ gas until the flask reached an initial differential pressure of $0.370 \mathrm{~atm}$. The pressure in the flask was measured by a differential pressure manometer (Extech Instrument, Model \#407910), and for all hydrogenation reactions the same initial pressure was maintained. The AuPd nanotriangle solution was then stirred under hydrogen for 30 minutes. Then 2-methyl-3buten-2-ol $(0.10 \mathrm{~mL})$ was injected into the flask. Similar to the cross-coupling reactions, hydrogenation reactions were performed in both light and dark conditions. For the ${ }^{1} \mathrm{H}$ NMR analysis, an aliquot of $100 \mu \mathrm{L}$ of the reaction mixture was collected every 20 minutes and was directly extracted in $500 \mu \mathrm{L}$ $\mathrm{CDCl}_{3}$.

\section{Results and discussion}

\section{AuPd bimetallic nanotriangles}

$\mathrm{Au}$ nanotriangles were synthesized via a literature procedure, which is a competitive process involving both reduction/ oxidative etching of $\mathrm{Au}$ in the presence of CTAC as a stabilizing agent. ${ }^{40}$ Here, ascorbic acid was used as the reducing agent and potassium iodide as an oxidative etchant in the presence of air. To synthesize the AuPd bimetallic nanotriangles, $\mathrm{K}_{2} \mathrm{PdCl}_{4}$ was reduced onto the as-synthesized $\mathrm{Au}$ nanotriangles using ascorbic acid as the reducing agent. These $\mathrm{Au}$ and AuPd nanotriangles were then characterized by UV-vis spectroscopy and TEM; the results are shown in Fig. 1. In the UV-vis spectrum, the Au nanotriangles show a broad plasmon peak at $605 \mathrm{~nm}$ with a shoulder at $534 \mathrm{~nm}$. The plasmon peak at $605 \mathrm{~nm}$ is attributed to the in-plane dipole mode, ${ }^{41}$ while the shoulder at $534 \mathrm{~nm}$ is likely due to the presence of spherical $\mathrm{Au}$ nanoparticles. ${ }^{2,40}$ Upon coating with Pd, a $c a .60 \mathrm{~nm}$ blue shift in the plasmon peak was observed. This might be due to some surface alloying between $\mathrm{Au}$ and Pd. ${ }^{42}$ The morphologies of the $\mathrm{Au}$ and AuPd nanotriangles were confirmed by TEM analysis (Fig. 1b-d). Fig. 1b shows the presence of a large population of $\mathrm{Au}$ nanotriangles; the average edge length of these nanotriangles was found to be $43 \pm 4 \mathrm{~nm}$. However, the sample also contains ca. $10 \%$ of quasi-spherical Au nanoparticles along with the nanotriangles. Presence of multiple shapes broadens the absorption spectrum; which leads to a good match with the incoming green LED spectrum. Fig. 1c shows a TEM image of the AuPd nanotriangles, and a higher resolution TEM image is shown in Fig. 1d. The Z-contrast difference in the TEM images of AuPd (Fig. 1c and d) indicates the spatial distribution of $\mathrm{Au}$ and Pd within the nanotriangle; the lighter regions are the $\mathrm{Pd}$ shell, and the darker region corresponds to the Au core. The Pd shells of the AuPd nanotriangles are polycrystalline, and thus, the resulting AuPd nanotriangles appear textured with unevenly distributed $\mathrm{Au}$ and $\mathrm{Pd}$ sites on their surface. A similar morphology was observed in AuPd nanowheels due to the Pd alloying with $\mathrm{Au}^{32}$ The morphology likely occurs due to the deposition of Pd at multiple sites on each particle, which could be due to defects in the CTAC coating on the surface of the Au nanotriangles. ${ }^{43}$ For catalytic purposes, the polycrystalline nature of the Pd shell may be favorable as the available surface area of the Pd catalyst would be substantially higher than a perfect epitaxial core-shell structure.

\section{Plasmon enhanced Suzuki-coupling reactions}

After confirming the morphology of the AuPd nanotriangles, they were used to catalyze Suzuki cross-coupling reactions. The reactions were performed both with and without light illumination. For the dark reactions, the reaction flasks were covered with aluminum foil; for the light reactions, the flasks were illuminated with a green LED (power $\sim 500 \mathrm{~mW}, \lambda_{\max }=530 \mathrm{~nm}$ ) light source (Fig. 2a). The green light source was chosen to match the wavelength $(534 \mathrm{~nm})$ of the AuPd plasmon band (Fig. 1a). At this wavelength, the surface plasmons of both the triangular and spherical AuPd nanoparticles will be excited. The light source setup is shown in Fig. 2a; it contains an array of 8 green LEDs mounted on each heat sink, and four of these heat sinks are assembled in such a way that a $25 \mathrm{~mL}$ flask can be placed in the center and receive $360^{\circ}$ illumination.

To investigate the plasmonic effect of AuPd nanotriangles on the Suzuki cross-coupling reaction, we chose to study a model reaction between $p$-iodobenzoic acid and phenylboronic acid, as shown in Scheme 1. This reaction was performed in both dark and light illumination, and the reaction kinetics were monitored by ${ }^{1} \mathrm{H}$ NMR. The percent conversions were calculated from the ${ }^{1} \mathrm{H}$ NMR spectra, and are plotted against time (Fig. 2b). The cross-coupling reaction performed in the light showed significantly higher conversions compared to the dark reactions. In both cases, only hetero-cross-coupling products were seen; i.e. no homocoupling products were observed. For example, after $1 \mathrm{~h}$ of reaction time, the light reaction had proceeded to $\sim 40 \%$ conversion, whereas the dark reaction had proceeded to only $\sim 23 \%$ conversion. With an increase in time, the difference in conversion between the light and dark reactions was more 
(a)

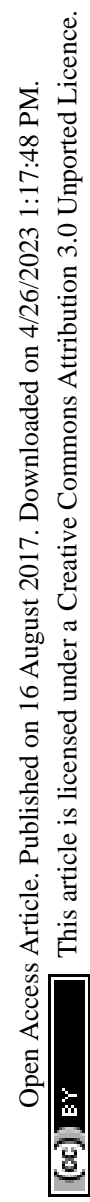
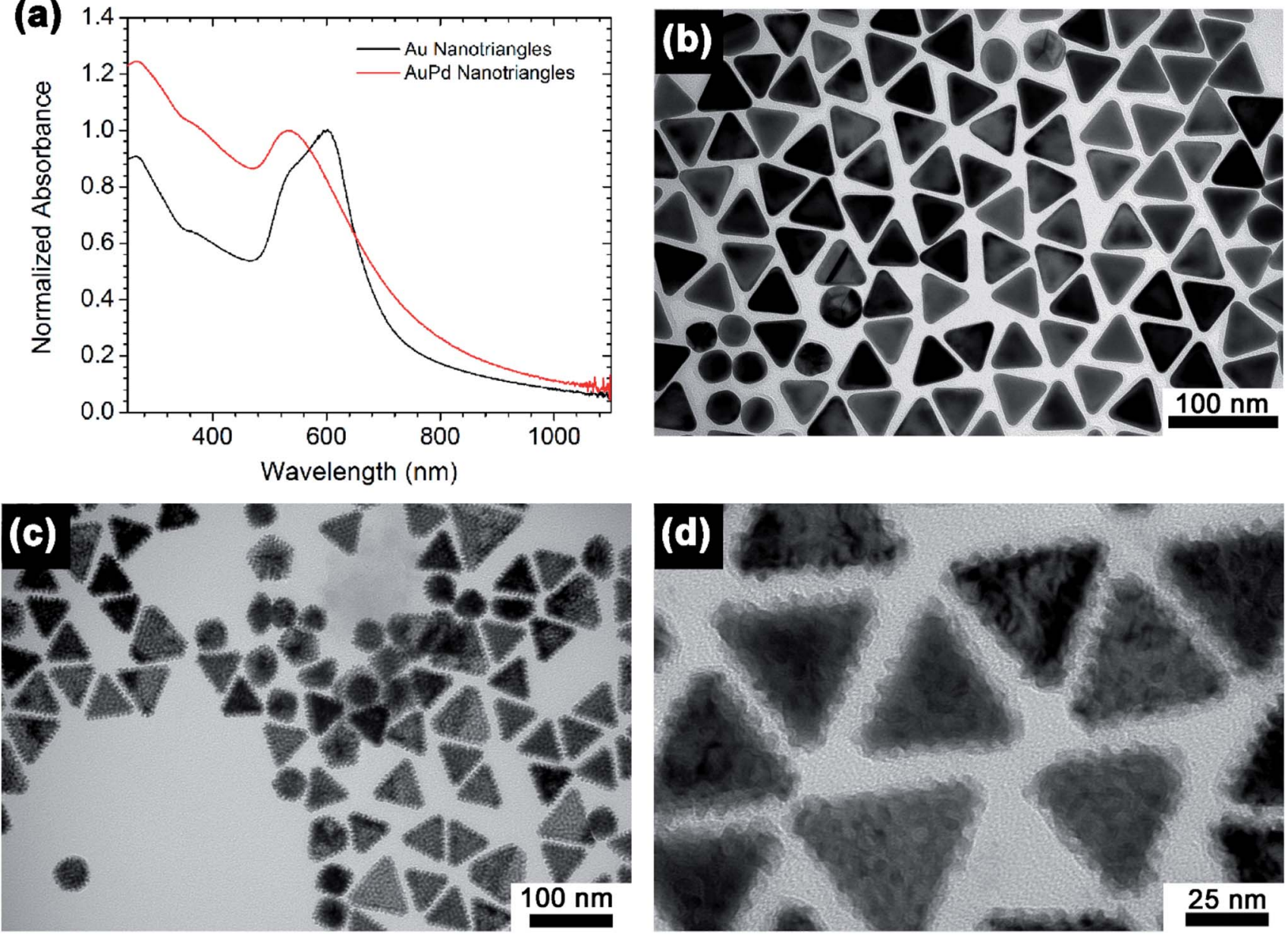

Fig. 1 (a) Normalized UV-vis spectra of Au and AuPd bimetallic nanotriangles; the spectra are normalized to the maximum of the plasmon band. TEM images of (b) as-synthesized Au nanotriangles, (c) AuPd bimetallic nanotriangles and (d) magnified image of AuPd nanotriangles.

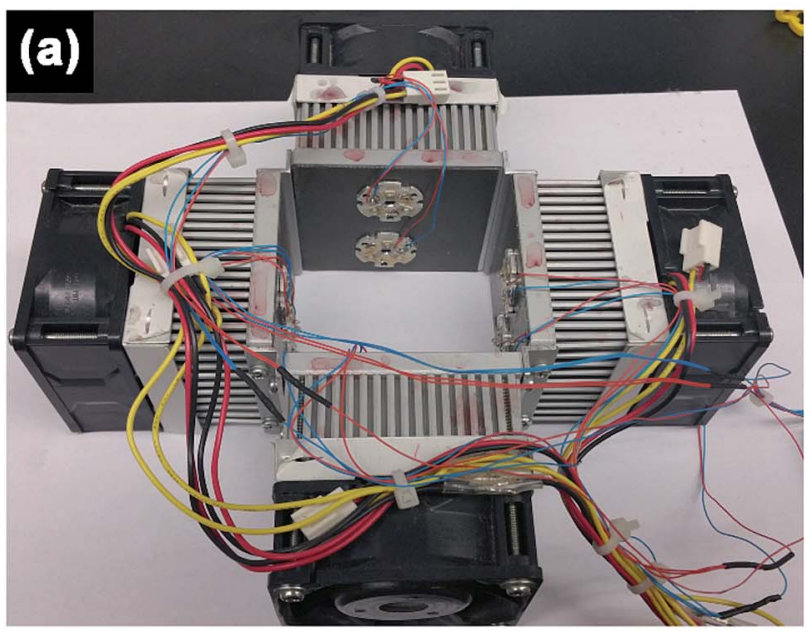

(b)

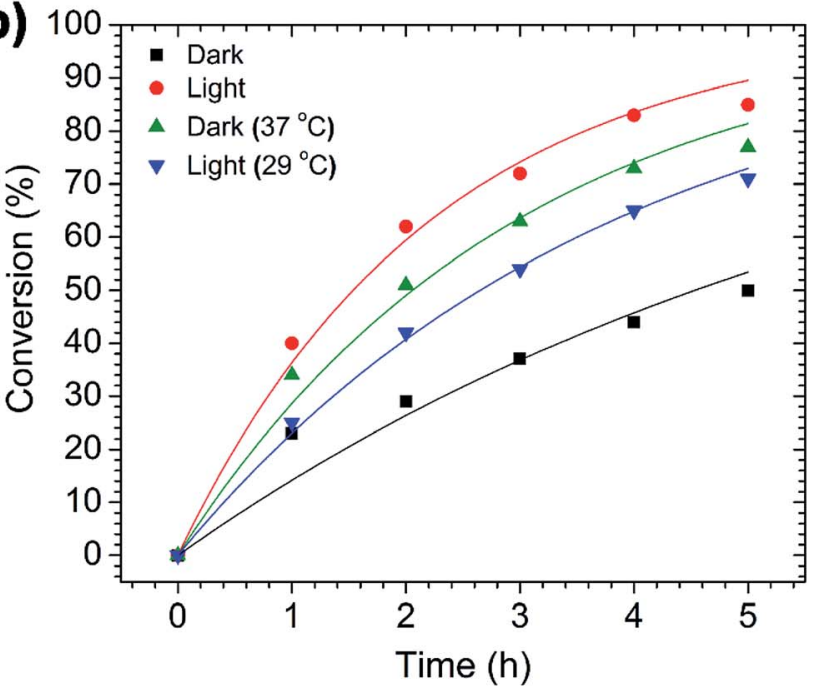

Fig. 2 (a) The LED light source with an array of green LEDs mounted on heat sinks, (b) percent conversions calculated from ${ }^{1} \mathrm{H}$ NMR are plotted against time; the reaction is between $p$-iodobenzoic acid and phenylboronic acid. The green triangles indicate the control dark reaction performed in a hot water bath and the inverted blue triangles indicate the light reaction performed in a $25^{\circ} \mathrm{C}$ water bath. The solid lines are a best-fit using a first-order kinetic model. 


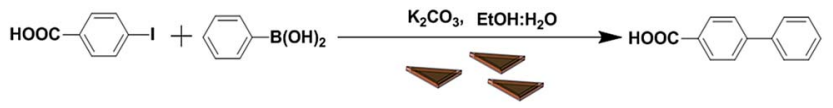

Scheme 1 The model Suzuki reaction using AuPd nanotriangles as catalysts.

pronounced. After $5 \mathrm{~h}$ of the reaction time, the light reaction showed $c a .35 \%$ higher conversion compared to the dark. At the same time, the temperature of both the reaction mixtures was measured. We noticed a rise in the final temperature of the light reaction from $25{ }^{\circ} \mathrm{C}$ (the initial temperature of the reaction flask) to $37^{\circ} \mathrm{C}$, while the dark reaction showed no change in temperature. To determine if this increase in temperature was due to the presence of AuPd nanotriangles or due to lightinduced heating of the solvent, we illuminated water with and without AuPd nanotriangles. After illuminating both the flasks for $1 \mathrm{~h}$, an increase in temperature from $25^{\circ} \mathrm{C}$ to $37^{\circ} \mathrm{C}$ for the flask containing AuPd nanotriangles was observed, whereas the flask containing only water showed only a $2{ }^{\circ} \mathrm{C}$ (light-induced heating) rise in temperature. This clearly confirms that the AuPd nanotriangles are the cause of the temperature increase; this is due to the plasmonic local heating generated by nonradiative plasmon decay (electron-phonon scattering). ${ }^{31}$

To determine how significant a role this plasmonic heating effect has on the overall enhancement of the reaction rate, we performed a control reaction in the dark using a hot water bath to maintain the temperature of the reaction mixture at $37^{\circ} \mathrm{C}$ (the same temperature reached by the light reaction flask). The reaction progress was monitored hourly by ${ }^{1} \mathrm{H}$ NMR, and the conversion plotted in Fig. 2b. The light reaction still showed higher conversions compared to the dark reaction run at $37^{\circ} \mathrm{C}$, although the final temperatures of both the reaction mixtures were same $\left(37^{\circ} \mathrm{C}\right)$. However, the difference in conversion is only $9 \%$ after $5 \mathrm{~h}$ of the reaction time; this is much lower compared to the previous case (35\%). From these results, it is clear that the rate of reaction is accelerated by the excitation of the AuPd surface plasmon modes. The effect of plasmonic excitation is also similar to that of conventional heating in improving the overall rate. To further support these conclusions, another Suzuki reaction was performed in which the temperature of the light reaction flask was fixed at $25^{\circ} \mathrm{C}$ using a water bath. After $5 \mathrm{~h}$, this reaction showed significantly higher conversion (ca. 20\%) than the dark reaction. However, the final temperature increased to $29^{\circ} \mathrm{C}$ (even though the reaction flask was kept in a $25{ }^{\circ} \mathrm{C}$ water bath). This slight rise in temperature may be due to the direct photothermal heating by both solvent and the water bath, or simply an insufficiently large thermal mass in the cooling bath. A number of other papers have outlined a similar role of plasmonic heating for enhancing the rate of a variety of catalytic reactions. ${ }^{21,29,30,44,45}$

However, in addition to the plasmonic heating effect, many reports have observed that plasmonic hot electron transfer contributes to the rate of a cross-coupling reaction. ${ }^{16,17}$ In many of these reports, a high power laser source was used to excite the $\mathrm{Au}$ nanoparticle surface plasmons, whereas, in the current study, we used a relatively low power $(\sim 500 \mathrm{~mW})$ and unfocused LED light source. The generation of plasmonic hot electrons is highly dependent on the power of the excitation light source..$^{45,46}$ The low power LEDs used here are likely insufficient to produce a significant population of hot electrons; hence, the contribution of these hot electrons in improving the overall rate of the reaction is low. Nonetheless, the dark control reactions underperformed the light reactions even when both were run at the same temperature. It is therefore possible that there is a minor contribution from plasmonic hot electron transfer to the overall enhancement.

We extended our studies to examine the effect of different aryl halides and different boronic acids. The results for various substrates are shown in Table 1. Selectivity for the heterocoupling product was $100 \%$ in all cases (though, for entry 2 and 3 both reactions would have the same product). In all cases, the reactions performed in the light showed an average of $\sim 20 \%$ higher conversion compared to those carried out in the dark. A large difference in the conversions between the light and dark reactions was observed when aromatic iodides were used as substrates. This is particularly seen for entry 4 , where a nitrosubstituted aryl iodide ( $p$-iodonitrobenzene) was reacted with phenylboronic acid. This difference was slightly higher even when compared to the other reactions where other aryl iodides were reacted with the same boronic acid (entries 1 and 2). The light reaction in entry 4 showed complete conversion, and the dark reaction showed a decreased conversion compared to entries 1 and 2. The presence of an electron-withdrawing group such as $-\mathrm{NO}_{2}$ on an aryl halide generally improves the rate of oxidative addition (typically the rate determining step) in Suzuki reactions. It is possible that the further acceleration of the reaction in the light could be coming from plasmonic hot electron transfer. However, the exact reason why the nitro-group shows a positive effect on the light reaction and a negative effect on the dark reaction is unclear. On the other hand, the difference in conversions was lower when the same halide ( $p$-iodonitrobenzene) was reacted with methyl-substituted phenylboronic acid (entry 7); the difference in conversion between light and dark was very low. Here, due to the presence of an electron-donating methyl group on the boronic acid, both the light and dark reactions showed almost complete conversions. Interestingly, in the case of entry 5 , where 2-thiopheneboronic acid was used, the reaction showed almost double the conversion in the presence of light as opposed to the dark. Generally, sulfur-containing (thiophene-based) substrates have been shown to poison the Pd catalyst and cause low yields in cross-coupling reactions. ${ }^{47}$ They often need either high catalyst loadings or elevated temperatures to achieve good yields. In such reactions, the use of plasmonic nanoparticles might be a promising way of improving the overall conversions, while keeping the catalyst loading to a minimum. Finally, among all of the reactions, the aryl bromide substrate (entry 3) showed the lowest overall conversion, regardless of whether or not the reaction was illuminated. This can be attributed to the poor leaving ability of $\mathrm{Br}$ as compared to $\mathrm{I}$.

\section{Catalyst stability and recyclability}

The main advantage of employing nanoparticles in catalysis is that the catalyst can easily be separated from the reaction 
Table 1 Percent conversions measured by ${ }^{1} \mathrm{H}$ NMR for different Suzuki reactions performed in both the dark and the light ${ }^{a}$

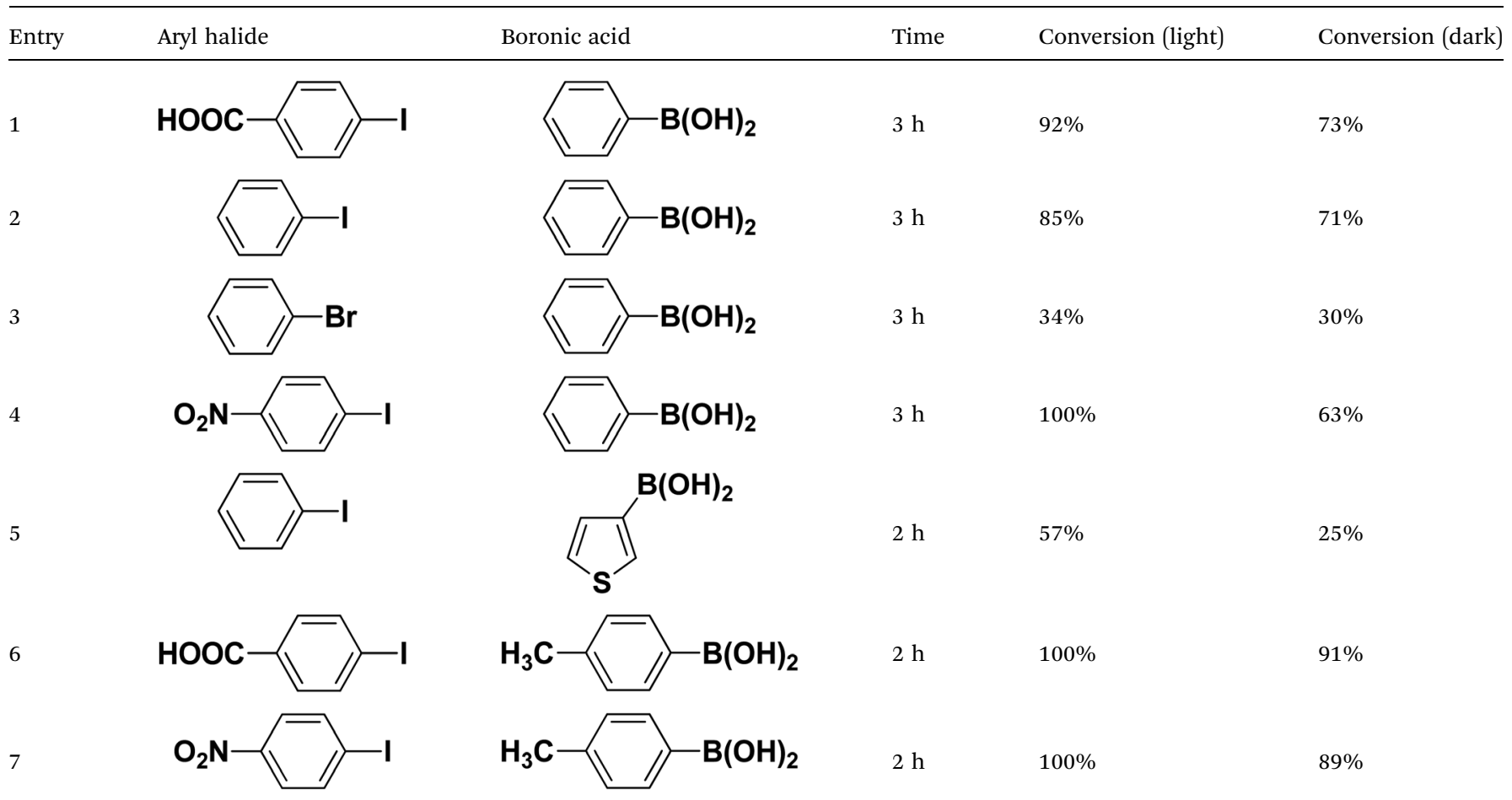

\footnotetext{
${ }^{a}$ The substrate to catalyst loading ratio for all reactions was $2.5: 0.002$. The initial reaction temperature was $25{ }^{\circ} \mathrm{C}$, and the solvent was a $80: 20$ mixture of water and ethanol.
}

mixture and re-used for several reaction cycles. To test the recyclability of the AuPd nanotriangles, we first characterized them after a Suzuki coupling reaction between $p$-iodobenzoic acid and phenylboronic acid. Fig. 3a shows the UV-vis spectra of the AuPd nanotriangles before and after the Suzuki reaction. The spectra were recorded for the AuPd nanotriangles used in the model cross-coupling reactions shown in Fig. 2b. After completion of the reaction, the AuPd nanotriangles were collected by centrifuging the reaction mixture, and then redispersed in a volume of water equivalent to the initial volume of the reaction mixture. A slight decrease in the intensity of the plasmon peak at $532 \mathrm{~nm}$ was observed after the reaction (Fig. 3a). This suggests a slight decrease in the population of AuPd nanotriangles after the reaction. However, an increase in the optical density was observed in the wavelength range from 200-300 nm after one reaction cycle. This might be due to the Rayleigh scattering from large aggregates in the reaction mixture. From TEM analysis (Fig. 3c), aggregation of some of the nanotriangles was evidenced after one reaction cycle. As a result of the aggregation, the nanotriangles tended to settle at the bottom of the reaction flask upon keeping the flask undisturbed for several days. We are uncertain as to why aggregation might be occurring in this system, but we believe it is due to the loss of their surface stabilizer (CTAC) during the Suzuki coupling reaction. A control experiment showed that the aggregation is not light-induced, and no such aggregation was seen for the hydrogenation system discussed below.
Using the same reactants that were used in the model Suzuki reaction between $p$-iodobenzoic acid and phenylboronic acid, we studied the catalyst recyclability over three reaction cycles in the presence of light. After each cycle, the AuPd nanotriangles were purified by centrifugation and then reused by redispersing them in the same amount of the reaction mixture. After $3 \mathrm{~h}$ of reaction, the percent conversion was calculated from the ${ }^{1} \mathrm{H}$ NMR spectrum, and the data is plotted against cycle number (Fig. 3b). In cycle 1, after $3 \mathrm{~h}$ of reaction, $\mathrm{ca}$. $70 \%$ conversion was observed, and the final temperature of the reaction flask was $37{ }^{\circ} \mathrm{C}$. In cycle 2 , the final temperature decreased slightly to $35{ }^{\circ} \mathrm{C}$, and the percent conversion was reduced to $c a .50 \%$. The decrease in conversion may be due to the decrease in the number of available active sites, since the nanoparticles are aggregated after the reaction (Fig. 3c). In the $3^{\text {rd }}$ cycle, the reaction yielded only $1 \%$ conversion and a final temperature of $31{ }^{\circ} \mathrm{C}$; this could be due to almost complete aggregation of the nanotriangles as the color of the solution substantially became lighter after redispersing the nanotriangles for the $3^{\text {nd }}$ reaction cycle. However, stability and hence catalytic recyclability of these nanoparticles may be further improved by either integrating them in porous metal oxide matrix ${ }^{48,49}$ or by coating them with thin shells. ${ }^{50}$

\section{Plasmon enhanced hydrogenation reaction}

To determine if the plasmonic properties of AuPd nanotriangles can be used to drive other Pd-catalyzed reactions, we studied the 
(a)

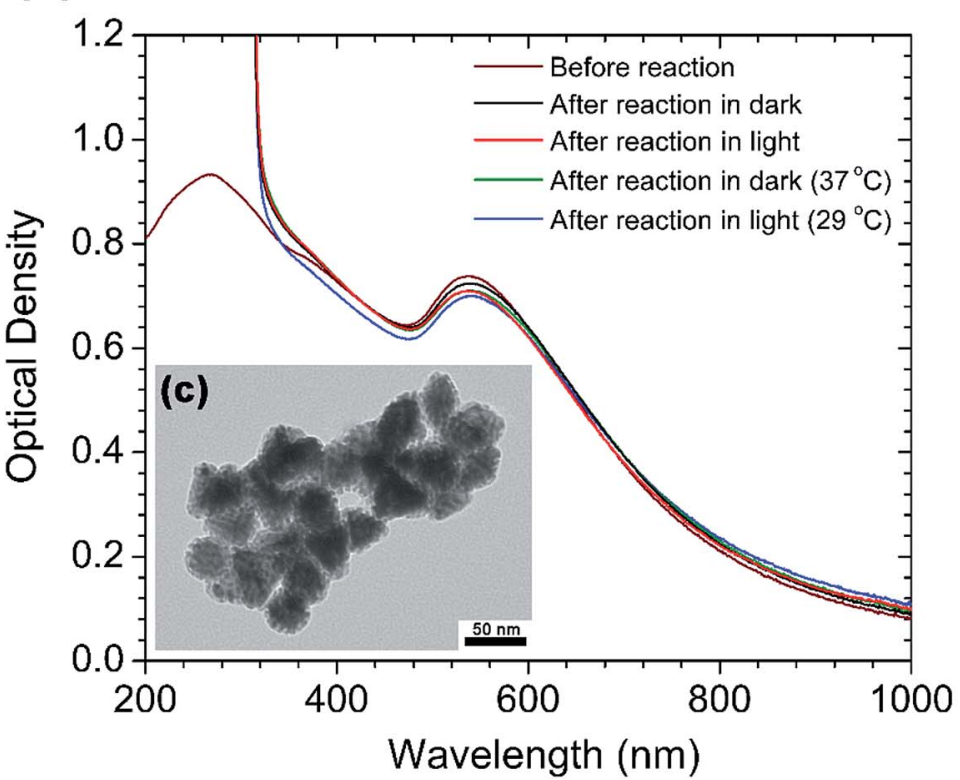

(b)

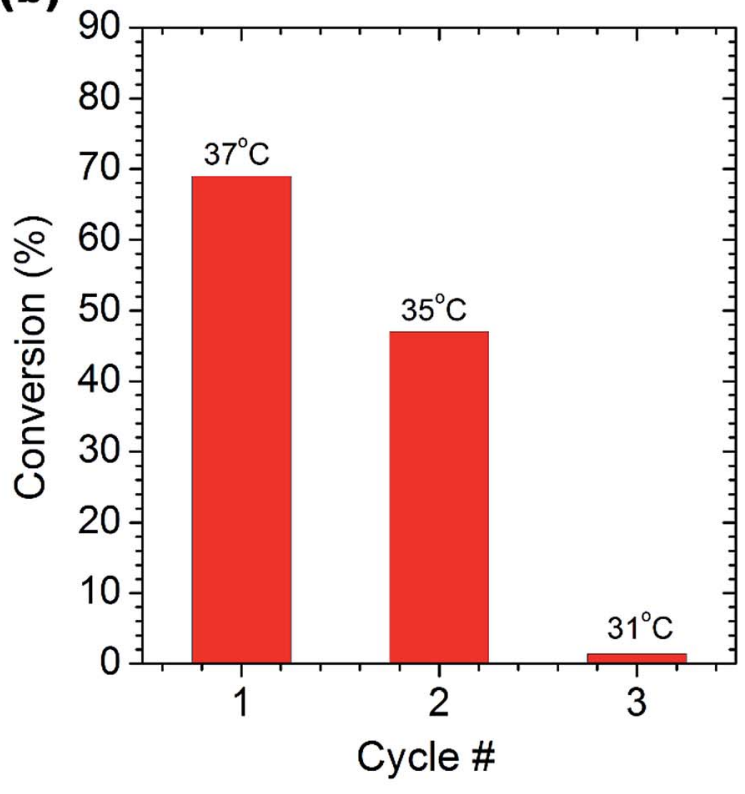

Fig. 3 (a) UV-vis absorption spectra of AuPd nanotriangles before and after cross-coupling reactions performed between $p$-iodobenzoic acid and phenylboronic acid. (b) Recyclability of AuPd nanotriangles and final temperature of the same coupling reaction performed under light illumination, and (c) TEM image showing aggregation of the AuPd nanotriangles, recorded after one cycle of the light reaction.

hydrogenation of 2-methyl-3-buten-2-ol (MBE) using the nanotriangles as the catalyst. The reactions were performed in both the presence and absence of light illumination. We used the same LED source (Fig. 2a) to irradiate the reaction mixture in light reaction. Here, we used a larger $(1 \mathrm{~L})$ round-bottom flask to maintain an excess of $\mathrm{H}_{2}$ gas for the hydrogenation reaction. The AuPd nanotriangles were added to the flask, and an excess of $\mathrm{H}_{2}$ gas was then supplied. Before injecting the substrate (MBE) into the flask, the flask was stirred to homogenize the solution. At $20 \mathrm{~min}$ intervals, the reaction was sampled and analyzed by ${ }^{1} \mathrm{H}$ NMR, and the percent conversions are plotted against time (Fig. 4). At each time interval, the light reaction showed significantly higher conversions compared to the dark reaction. For example, after $20 \mathrm{~min}$, the light reaction had proceeded to $42 \%$ conversion, whereas the dark reaction showed only $18 \%$ conversion. The difference became more pronounced as the reaction proceeded. Similar to the plasmon enhanced Suzuki reactions, an increase in the temperature of the light reaction (from $23{ }^{\circ} \mathrm{C}$ to $30^{\circ} \mathrm{C}$ ) was observed. However, the magnitude of the temperature increase was not as significant as in the Suzuki reactions. This is most likely due to the slightly lower AuPd catalyst loading, which was nearly 4 times lower compared to the Suzuki reaction. Again, to understand the role of plasmonic heating in the enhancement of the hydrogenation reaction, a control dark reaction was performed by heating the flask to $30{ }^{\circ} \mathrm{C}$ in a conventional hot water bath (i.e., the same temperature reached by the light reaction). After 120 min of reaction time, the control dark reaction showed almost same conversion as the light reaction (Fig. 4). This is in contrast to the Suzuki reactions, where the light reaction showed slightly higher conversions ( $9 \%)$ compared to the control dark reaction (Fig. 2b). The fact that similar conversions are observed in both the reactions (light and dark at $30^{\circ} \mathrm{C}$ ) clearly suggests that the enhancement is due to plasmonic heating effects alone. If other plasmonic effects contributed significantly to the enhancement, the light reaction would show higher conversions compared to the high-temperature dark

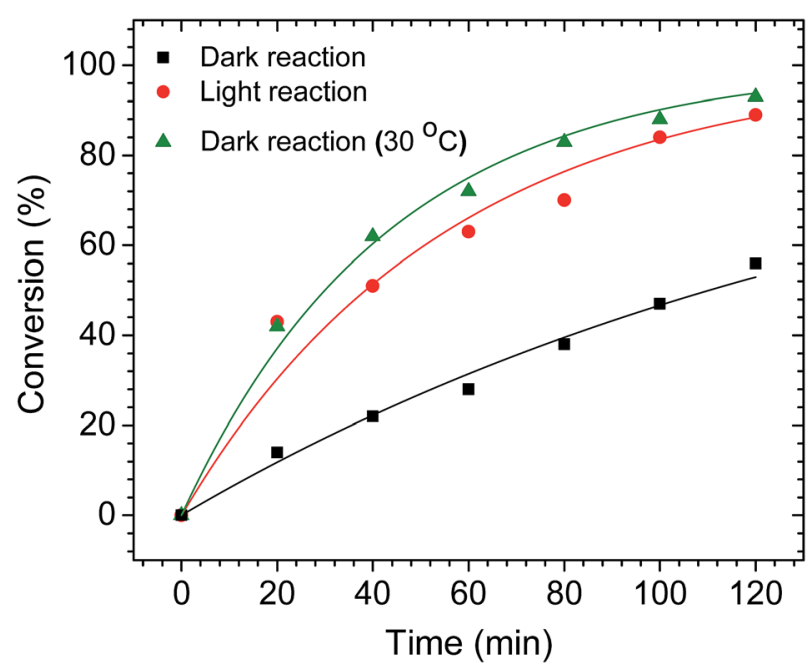

Fig. 4 Percent conversion of hydrogenation reactions performed on 2-methyl-3-buten-2-ol using AuPd nanotriangles in the presence and absence of light. The black squares show the reaction performed at $23{ }^{\circ} \mathrm{C}$ under ambient conditions, the red circles show the reaction performed under illumination that achieved a final temperature of $30^{\circ} \mathrm{C}$, and the green triangles show the dark reaction performed at $30{ }^{\circ} \mathrm{C}$ using hot water bath. The solid lines are a best-fit using a firstorder kinetic model. 
reaction. Similar results have been reported in the literature; ${ }^{\mathbf{2 1 , 5 1}}$ Long et al., observed the contribution of only plasmonic heating effect in the hydrogenation of styrene. ${ }^{51}$ Since it has been shown that $\mathrm{Au}$ nanoparticles alone can catalyze the hydrogenation reaction (although it requires high temperatures), ${ }^{46,52}$ we also attempted to perform the same hydrogenation reaction using pure Au nanotriangles as the catalyst. No product formation was observed in either the light or dark conditions. This confirmed that the Pd is the active site on the AuPd catalyst for the hydrogenation reactions. Another possible mechanism to drive the hydrogenation reactions is by plasmonic hot electron transfer; however, to achieve the dissociation of $\mathrm{H}_{2}$ by hot electrons generated on $\mathrm{Au}$, a high power incoming light source (on order of $\mathrm{W} \mathrm{cm}^{-2}$ ) is required. ${ }^{46}$ The LED light source used in the current study was likely not intense enough to produce sufficiently hot electrons, and no activity of Au was seen for this hydrogenation reaction. This result agrees with earlier crosscoupling and hydrogenation results that showed that photothermal heating is mostly responsible for the catalytic enhancement. Again, we note that this is a consequence of the low power LED source used in this study.

\section{Conclusions}

In conclusion, we have synthesized triangular AuPd nanoparticles and employed them to improve the rates of Suzuki coupling and hydrogenation reactions. Upon exciting the surface plasmons in AuPd nanotriangles using green LEDs (whose wavelength was near the maximum of the plasmon band), an improvement in the rates of Suzuki and hydrogenation reactions was observed. Our results revealed that the enhancement in the rate of reaction is mainly due to the contribution of plasmonic heating effects, with at most a minor contribution from plasmonic hot electron transfer. We have investigated the recyclability of these catalysts; we found that the catalyst survived for 2 cycles of Suzuki reactions. TEM showed aggregation of the AuPd nanotriangles causing a reduction in the catalytic activity. This study clearly demonstrates that a plasmonic enhancement in the rates of Pdcatalyzed reactions can be observed even using a low power and unfocused LED light source, and that, under these low powers, there is only a plasmonic photothermal effect contributing to the rate enhancement.

\section{Conflicts of interest}

There are no conflicts to declare.

\section{Acknowledgements}

T. L. K. is a Canada Research Chair in Photovoltaics. This research was undertaken, in part, thanks to funding from the Canada Research Chairs program. A. M. F. thanks the Coordination for the Improvement of Higher Level Personnel-CAPES for a scholarship (doctoral sandwich grant, PDSE 99999.009761/2014-04). The Natural Sciences and Engineering
Research Council of Canada (NSERC) and the University of Saskatchewan are acknowledged for financial support.

\section{References}

1 M. K. Gangishetty, K. E. Lee, R. W. J. Scott and T. L. Kelly, ACS Appl. Mater. Interfaces, 2013, 5, 11044-11051.

2 M. K. Gangishetty, R. W. J. Scott and T. L. Kelly, Langmuir, 2014, 30, 14352-14359.

3 H. A. Atwater and A. Polman, Nat. Mater., 2010, 9, 205-213.

4 C. Clavero, Nat. Photonics, 2014, 8, 95-103.

5 J. N. Anker, W. P. Hall, O. Lyandres, N. C. Shah, J. Zhao and R. P. Van Duyne, Nat. Mater., 2008, 7, 442-453.

6 S. Linic, U. Aslam, C. Boerigter and M. Morabito, Nat. Mater., 2015, 14, 567-576.

7 L. Guerrini and D. Graham, Chem. Soc. Rev., 2012, 41, 70857107.

8 Z. Lou, Q. Gu, Y. Liao, S. Yu and C. Xue, Appl. Catal., B, 2016, 184, 258-263.

9 E. L. Runnerstrom, A. Bergerud, A. Agrawal, R. W. Johns, C. J. Dahlman, A. Singh, S. M. Selbach and D. J. Milliron, Nano Lett., 2016, 16, 3390-3398.

10 K. L. Kelly, E. Coronado, L. L. Zhao and G. C. Schatz, J. Phys. Chem. C, 2002, 107, 668-677.

11 M. J. Kale, T. Avanesian and P. Christopher, ACS Catal., 2014, 4, 116-128.

12 P. Wang, B. Huang, Y. Dai and M.-H. Whangbo, Phys. Chem. Chem. Phys., 2012, 14, 9813-9825.

13 R. Jiang, B. Li, C. Fang and J. Wang, Adv. Mater., 2014, 26, 5274-5309.

14 J. Li, S. K. Cushing, J. Bright, F. Meng, T. R. Senty, P. Zheng, A. D. Bristow and N. Wu, ACS Catal., 2013, 3, 47-51.

15 L. Liu, S. Ouyang and J. Ye, Angew. Chem., Int. Ed., 2013, 125, 6821-6825.

16 Q. Xiao, S. Sarina, A. Bo, J. Jia, H. Liu, D. P. Arnold, Y. Huang, H. Wu and H. Zhu, ACS Catal., 2014, 4, 1725-1734.

17 F. Wang, C. Li, H. Chen, R. Jiang, L.-D. Sun, Q. Li, J. Wang, J. C. Yu and C.-H. Yan, J. Am. Chem. Soc., 2013, 135, 55885601.

18 Q. Xiao, S. Sarina, E. R. Waclawik, J. Jia, J. Chang, J. D. Riches, H. Wu, Z. Zheng and H. Zhu, ACS Catal., 2016, 6, 1744-1753.

19 L.-B. Zhao, M. Zhang, Y.-F. Huang, C. T. Williams, D.-Y. Wu, B. Ren and Z.-Q. Tian, J. Phys. Chem. Lett., 2014, 5, 12591266.

20 Z. Liu, Y. Huang, Q. Xiao and H. Zhu, Green Chem., 2016, 18, 817-825.

21 X. Zhao, R. Long, D. Liu, B. Luo and Y. Xiong, J. Mater. Chem. A, 2015, 3, 9390-9394.

22 J. Cui, Y. Li, L. Liu, L. Chen, J. Xu, J. Ma, G. Fang, E. Zhu, H. Wu, L. Zhao, L. Wang and Y. Huang, Nano Lett., 2015, 15, 6295-6301.

23 Q. Xiao, Z. Liu, A. Bo, S. Zavahir, S. Sarina, S. Bottle, J. D. Riches and H. Zhu, J. Am. Chem. Soc., 2015, 137, 1956-1966.

24 T. Jiang, C. Jia, L. Zhang, S. He, Y. Sang, H. Li, Y. Li, X. Xu and H. Liu, Nanoscale, 2015, 7, 209-217. 
25 S. Linic, P. Christopher and D. B. Ingram, Nat. Mater., 2011, 10, 911-921.

26 S. Sarina, H. Zhu, E. Jaatinen, Q. Xiao, H. Liu, J. Jia, C. Chen and J. Zhao, J. Am. Chem. Soc., 2013, 135, 5793-5801.

27 M. L. Brongersma, N. J. Halas and P. Nordlander, Nat. Nanotechnol., 2015, 10, 25-34.

28 W. R. Erwin, H. F. Zarick, E. M. Talbert and R. Bardhan, Energy Environ. Sci., 2016, 9, 1577-1601.

29 C. Wang, O. Ranasingha, S. Natesakhawat, P. R. Ohodnicki, M. Andio, J. P. Lewis and C. Matranga, Nanoscale, 2013, 5, 6968-6974.

30 G. K. Larsen, W. Farr and S. E. Hunyadi Murph, J. Phys. Chem. C, 2016, 120, 15162-15172.

31 J. R. Adleman, D. A. Boyd, D. G. Goodwin and D. Psaltis, Nano Lett., 2009, 9, 4417-4423.

32 X. Huang, Y. Li, Y. Chen, H. Zhou, X. Duan and Y. Huang, Angew. Chem., Int. Ed., 2013, 52, 6063-6067.

33 Q. Xiao, S. Sarina, E. Jaatinen, J. Jia, D. P. Arnold, H. Liu and H. Zhu, Green Chem., 2014, 16, 4272-4285.

34 P. Verma, Y. Kuwahara, K. Mori and H. Yamashita, J. Mater. Chem. A, 2016, 4, 10142-10150.

35 Z. Zheng, T. Tachikawa and T. Majima, J. Am. Chem. Soc., 2015, 137, 948-957.

36 M. Wen, S. Takakura, K. Fuku, K. Mori and H. Yamashita, Catal. Today, 2015, 242, 381-385.

37 S. Zhang, C. Chang, Z. Huang, Y. Ma, W. Gao, J. Li and Y. Qu, ACS Catal., 2015, 5, 6481-6488.

38 E. Hao and G. C. Schatz, J. Chem. Phys., 2004, 120, 357-366.
39 K. L. Kelly, E. Coronado, L. L. Zhao and G. C. Schatz, J. Phys. Chem. C, 2003, 107, 668-677.

40 L. Chen, F. Ji, Y. Xu, L. He, Y. Mi, F. Bao, B. Sun, X. Zhang and Q. Zhang, Nano Lett., 2014, 14, 7201-7206.

41 J. E. Millstone, S. Park, K. L. Shuford, L. Qin, G. C. Schatz and C. A. Mirkin, J. Am. Chem. Soc., 2005, 127, 5312-5313.

42 Y. Ding, F. Fan, Z. Tian and Z. L. Wang, J. Am. Chem. Soc., 2010, 132, 12480-12486.

43 M. Jaschke, H. J. Butt, H. E. Gaub and S. Manne, Langmuir, 1997, 13, 1381-1384.

44 T. Bora, D. Zoepfl and J. Dutta, Sci. Rep., 2016, 6, 26913.

45 P. Dombi, A. Hörl, P. Rácz, I. Márton, A. Trügler, J. R. Krenn and U. Hohenester, Nano Lett., 2013, 13, 674-678.

46 S. Mukherjee, F. Libisch, N. Large, O. Neumann, L. V. Brown, J. Cheng, J. B. Lassiter, E. A. Carter, P. Nordlander and N. J. Halas, Nano Lett., 2013, 13, 240-247.

47 Y. Li, X. M. Hong, D. M. Collard and M. A. El-Sayed, Org. Lett., 2000, 2, 2385-2388.

48 D. Han, Z. Bao, H. Xing, Y. Yang, Q. Ren and Z. Zhang, Nanoscale, 2017, 9, 6026-6032.

49 A. Samanta, T. Rajesh and R. Nandini Devi, J. Mater. Chem. A, 2014, 2, 4398-4405.

50 Y. Mu, H. Zhang, W. Zheng and X. Cui, New J. Chem., 2017, 41, 786-792.

51 R. Long, Z. Rao, K. Mao, Y. Li, C. Zhang, Q. Liu, C. Wang, Z.-Y. Li, X. Wu and Y. Xiong, Angew. Chem., Int. Ed., 2015, 54, 2425-2430.

52 T. Mitsudome and K. Kaneda, Green Chem., 2013, 15, 26362654. 